\title{
Selection and Mass Production of Santalum Album L. Calli for Induction of Sesquiterpenes
}

\author{
Julien Crovadore, Michel Schalk \& François Lefort
}

To cite this article: Julien Crovadore, Michel Schalk \& François Lefort (2012) Selection and Mass Production of Santalum Album L. Calli for Induction of Sesquiterpenes, Biotechnology \& Biotechnological Equipment, 26:2, 2870-2874, DOI: 10.5504/BBEQ.2012.0028

To link to this article: https://doi.org/10.5504/BBEQ.2012.0028

\section{(c) 2012 Taylor and Francis Group, LLC}

\section{曲 Published online: 16 Apr 2014.}

Submit your article to this journal $₫$

III Article views: 744

Q View related articles ¿

4 Citing articles: 9 View citing articles 


\title{
SELECTION AND MASS PRODUCTION OF SANTALUM ALBUM L. CALLI FOR INDUCTION OF SESQUITERPENES
}

\author{
Julien Crovadore ${ }^{1}$, Michel Schalk ${ }^{2}$ and François Lefort ${ }^{1}$ \\ ${ }^{1}$ University of Applied Sciences of Western Switzerland, Geneva Institute of Technology, Architecture and Landscape, Research \\ Institute Earth Nature and Environment, Jussy, Switzerland \\ ${ }^{2}$ Firmenich SA, Biotechnology Department, Corporate R\&D Division, Geneva, Switzerland \\ Corresponding author: François Lefort \\ E-mail: francois.lefort@hesge.ch
}

\begin{abstract}
Young hypocotyl segments obtained from aseptically germinated seeds of Santalum album L. (5 weeks old) were used to induce callus formation. Growth of different calli types was induced on hypocotyls segments under different conditions. Resulting green calli were maintained on MS medium and Gamborg basal medium supplemented with 2,4D (2,4-Dichlorophenoxyacetic acid) $(0.5 \mu M)$ and Kin (Kinetin) $(10 \mu M)$. Numerous concentrations of 2,4D and Kin were tested in combination with inducers of the sesquiterpene metabolism and one promising callus type was selected for mass production. This interesting type of callus has been used for searching functional genes in the biosynthetic pathway leading to sesquiterpenes molecules.
\end{abstract}

Biotechnol. \& Biotechnol. Eq. 2012, 26(2), 2870-2874

Keywords: in vitro culture, sandalwood oil, Santalum album, sesquiterpene

Abbreviations: 2,4D: 2,4-Dichlorophenoxyacetic acid; ACC: 1-aminocyclopropane-1-carboxylic acid; Azacytidine: 4-amino-1- $\beta$-D-ribofuranosyl-1,3,5-triazin-2(1H)-one;

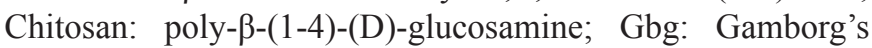
B-5 Basal medium with min. organics; Ethephon: 2-chloroethylphosphonic acid; Kin: Kinetin; MS: Murashige and Skoog medium; NAA: Naphthaleneacetic acid

\section{Introduction}

Santalum album L., most known as Indian sandalwood, is a root hemi-parasitic tree belonging to the family of the Santalaceae and renowned worldwide and particularly in the field of perfumery for the famous and unique oil extracted from its heartwood. This species was registered in 1998 in the vulnerable category of the IUCN (International Union for Conservation of Nature) red list (9), due to important diseases and overexploitation of the natural resources for the use in traditional medicine, oil extraction for perfume and for other uses such as carving, making furniture or religious ceremonies. The situation is similar for close species growing in the Pacific and Australasian regions such as Santalum austrocaledonicum, S. yasi and S. insulare, which are also considered as endangered by the CITES (Convention on International Trade in Endangered Species). The oil demand around the world is, at the contrary of the supply, ever increasing. Moreover, smuggling in the last decades has reached alarming proportions $(1,10)$. In this context, more and more investigations are carried out with the final objective of establishing sustainable production of sandalwood oil and sandalwood derived products. Because natural propagation in Santalum album and related species is slow and difficult, biotechnology has been considered as a valuable alternative. A key stage of such research is the ability to produce plants and biomass through micropropagation and in vitro cell culture. For the past 30 years, these aspects have provoked many studies $(3,4,11,13,14,15,16)$ and such research is still going on (17). The elucidation of the biosynthetic pathway leading to the sesquiterpenes molecules identified as key odorant in this material is of particular interest. Trade oils from S. album mainly contain santalols (50-70\%) associated with close metabolites (8), with $\alpha$-santalol ranging from $40 \%$ to $55 \%$ and $\beta$-santalol from $17 \%$ to $27 \%$ of total oil (20). From the two molecules $\beta$-santalol is the main source of the odour profile, while $\alpha$-santalol has a very weak odour. Degradative alcohols such as $\alpha$-santalal and other molecules also contribute to the odour profile described by Anonis (2).

The present work contributes to these efforts by reporting the production of a selected type of Santalum album callus that can be used for the elucidation of some secondary metabolic pathways for santalol production.

\section{Materials and Methods}

Santalum album L. seeds from Kununurra, WA, were provided by the Forest Products Commission (Seed Centre, locked bag 6, Manjimup, 6258 Western Australia, Australia).

Seeds were surface sterilized with sodium hypochlorite. Different concentrations of sodium hypochlorite $(0.5,1$ and $2.5 \%$ ) were applied for several treatment lengths: 20, 30, 40, 60, 90 and finally 120 minutes. Subsequently seeds were washed thrice in sterile ultrapure water $(20,10$ and 5 minutes, respectively). The surface sterilized seeds were scalpel shelled and placed on different culture media for germination in glass test tubes. The media for germination were Murashige-Skoog (MS; Sigma-Aldrich Co., Epalinges-Switzerland), MS1/2 and 
Gamborg's B-5 Basal medium with minimal organics (Gbg; Sigma-Aldrich Co.) $(7,12)$.

All media were supplemented with 3\% sucrose and solidified with Bactoagar ( $\left.7.8 \mathrm{~g} \cdot \mathrm{L}^{-1}\right)$ (Sigma, St. Louis, USA). The $\mathrm{pH}$ of media was adjusted to 5.7 prior to autoclaving at $121{ }^{\circ} \mathrm{C}$ for 20 minutes. Glass tubes were kept in culture room at $27^{\circ} \mathrm{C}$ under a $16 \mathrm{~h} \mathrm{light} / 8 \mathrm{~h}$ dark photoperiod with a light intensity of approximately 6000 lux emitted by cool white fluorescent (Sylvania LUXLINE PLUS F58W/840 - T8, 1500 $\mathrm{mm}$ ) and purple photosynthetic lamps (Sylvania GROLUX F58W/GRO, $1500 \mathrm{~mm}$ ).

Explants from different parts of plants obtained by in vitro germination were sampled: hypocotyls and crown sections (3$10 \mathrm{~mm}$ in length), cotyledon and leaf pieces $\left(10-16 \mathrm{~mm}^{2}\right.$ of surface area) and root segments (3-5 $\mathrm{mm}$ in length). Explants were taken from sterile seedlings of different stages (Fig. 1a) and were then placed in Petri dishes on MS and Gbg medium with various combinations of 2,4-dichlorophenoxyacetic acid (2,4D; Sigma-Aldrich Co.) and Kinetin (Kin; Sigma-Aldrich Co.) concentrations to induce callus growth.

The following range of Kin and 2,4D concentrations combinations were tested for callus initiation from Santalum album L. explants (Fig. 1c) in MS-based medium: MS 2,4D $(0.5 \mu \mathrm{M})+\operatorname{Kin}(0.5 \mu \mathrm{M})$; MS 2,4D $(0.5 \mu \mathrm{M})+\operatorname{Kin}(1 \mu \mathrm{M})$; MS 2,4D $(0.5 \mu \mathrm{M})+$ Kin $(10 \mu \mathrm{M})$; MS 2,4D $(4.5 \mu \mathrm{M})$ and finally MS Kin $(2.32 \mu \mathrm{M})$.

Resulting calli cultures were kept in culture rooms under the same temperature, light and photoperiod conditions described above for further transfer on inducing media for sesquiterpene synthesis.

Rooting was attempted starting from newly obtained shoots of Santalum album with different hormonal combinations on solid MS medium or by immersion in a naphthalene acetic acid (NAA; Sigma-Aldrich Co.) liquid medium as described in Sanjaya et al. (18).

Inducing of the sesquiterpene synthesis was carried out by transfer on Gbg culture media containing various concentrations of 2,4D, Kin combined with 1-aminocyclopropane-1-carboxylic acid (ACC), 2-chloroethylphosphonic acid (Ethephon), poly$\beta$-(1-4)-(D)-glucosamine (chitosan), salicylic acid, methyl jasmonate, L-methionine, coconut water, 4-amino-1- $\beta$-Dribofuranosyl-1,3,5-triazin-2(1H)-one (azacytidine). All chemicals were purchased by Sigma-Aldrich Co. (Epalinges, Switzerland).

The following concentration range of hormone combinations were tested for sesquiterpene induction in callus culture on media: Gbg 2,4D $(0.5 \mu \mathrm{M})+\mathrm{Kin}(0.5 \mu \mathrm{M})$; Gbg $2,4 \mathrm{D}(0.5 \mu \mathrm{M})+\mathrm{Kin}(1 \mu \mathrm{M}) ; \operatorname{Gbg} 2,4 \mathrm{D}(0.5 \mu \mathrm{M})+\mathrm{Kin}(10$ $\mu \mathrm{M})$; Gbg 2,4D $(4.5 \mu \mathrm{M})$ and finally Gbg Kin $(2.32 \mu \mathrm{M})$ in combination with a range of inducers. The following inducers at different concentrations were assayed: ACC $(0.1 \mathrm{mM}, 0.5$ $\mathrm{mM}, 1 \mathrm{mM}, 2 \mathrm{mM}, 5 \mathrm{mM})$, Ethephon $(5 \mu \mathrm{M}, 15 \mu \mathrm{M}, 25 \mu \mathrm{M}$, $100 \mu \mathrm{M})$, chitosan $\left(100 \mu \mathrm{g} \cdot \mathrm{L}^{-1}, 1 \mathrm{mg} \cdot \mathrm{L}^{-1}, 20 \mathrm{mg} \cdot \mathrm{L}^{-1}\right)$, salicylic acid $(10 \mu \mathrm{M}, 50 \mu \mathrm{M}, 200 \mu \mathrm{M})$, methyl jasmonate $(2 \mu \mathrm{M}, 10$ $\mu \mathrm{M}, 200 \mu \mathrm{M})$, L-methionine (1\%, 5\%, 10\%, 20\%), coconut water $(1 \%, 5 \%, 10 \%, 20 \%)$ and azacytidine $(0.3 \mathrm{mM}, 0.5 \mathrm{mM}$, $1 \mathrm{mM}, 2 \mathrm{mM}$ ). Finally all cultures were pursued on Gbg 2,4D $(0.5 \mu \mathrm{M})+\operatorname{Kin}(10 \mu \mathrm{M})$ which yielded the best callus growth rate.

Calli were sampled 2, 4, and 6 weeks, and in some experiments up to 14 weeks, after transfer on an inducing medium. The samples were extracted with pentane and analyzed by GC-MS on an Agilent 6890 Series GC system connected to an Agilent 5975 mass detector. The GC was equipped with $0.25 \mathrm{~mm}$ inner diameter by $30 \mathrm{~m}$ SPB-1 capillary column (Supelco, Bellefonte, PA). The carrier gas was He at a constant flow of $1 \mathrm{~mL} \cdot \mathrm{min}^{-1}$. The initial oven temperature was $80^{\circ} \mathrm{C}(1$ min hold) followed by a gradient of $10^{\circ} \mathrm{C} \cdot \mathrm{min}^{-1}$ to $300^{\circ} \mathrm{C}$. The identification of the products was based on the matching of the mass spectra and coincidence of the retention indices with data from Firmenich internal databases.

Genetic identity of plant material (leaves from grown plant and calli) was checked by PCR amplification and subsequent sequencing of the ITS region.

\section{Results and Discussion}

\section{Genetic identification}

PCR amplification from DNA extracted from leaves and calli yielded a unique sequence for the ITS region which was registered in GenBank under the accession number HM235968.

\section{In vitro production of Santalum album $\mathrm{L}$. seedlings}

The most crucial step in establishing in vitro plant tissue culture from sandalwood is the sterilisation of seeds. This was achieved by developing a protocol involving a very long seed disinfection period ( 2 hours) in a $2.5 \%$ sodium hypochlorite solution followed by 3 subsequent washings $(20,10$ and 5 minutes, respectively) in sterile water. The seeds were then carefully shelled with a scalpel, in sterile Petri dishes in sterile conditions under a laminar flow. This step was found to be mandatory for obtaining in vitro seed germination. Absolutely no germination could be obtained by skipping the shelling step. This step also allowed for selecting viable seeds based on their healthy appearance. Finally, the selected seeds were inoculated onto solid culture medium in glass tubes (Gamborg medium + Bactoagar $\left(7.8 \mathrm{~g} \cdot \mathrm{L}^{-1}\right)+$ sucrose $\left.(3 \%)\right)$ and germination occurred (Fig. 1a and Fig. 1b).

After a five-week culture period, an average rate of $95 \%$ healthy sandalwood seedlings was obtained. The germination rate reached $96 \%$ with an infection rate of $1 \%$. It seems that shelling the seeds brought a major advantage in comparison with the germination rates observed without shelling, whether it was in sterile culture conditions or in the green house. After germination, polyembryony was observed for a number of plants (ca. 2\%). These polyembryonic plants were discarded.

Some healthy seedlings obtained in vitro were also used in trials for acclimatization in pots in the glasshouse. Sandalwood seedlings were planted in $5 \mathrm{~L}$ pots (Klassmann 
2 substrate) with previously established two-year-old grafted Citrus plants. These trials were all successful and sandalwood plants established a root hemiparasitic relation with the Citrus vascular system. The implementation of this acclimatization protocol could be very useful in sandalwood planting programs.
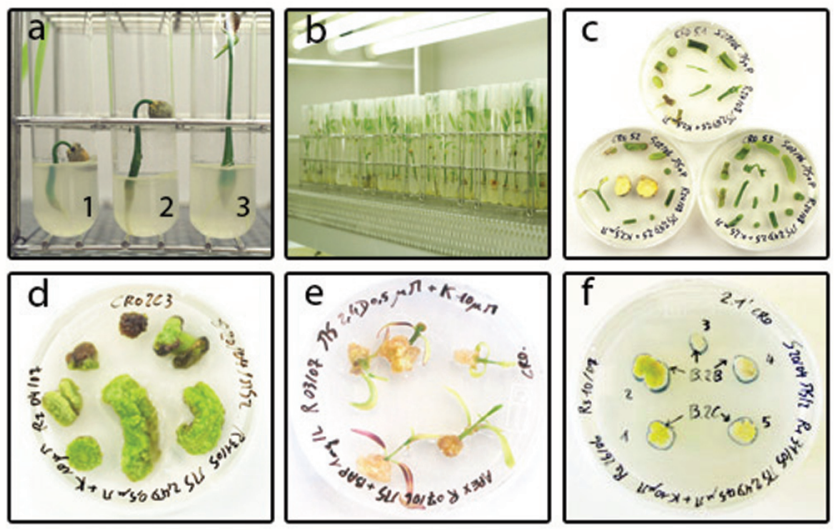

Fig. 1. Germination stages (a) - respectively, 5-day (1) and 10-day germinated seed (2), and visible epicotyl stage (3); Santalum album L. in vitro seedlings one month and a half old (b); explant reactivity trials (c); green and dense calli from hypocotyl segments (d); white and friable calli from seedling shoot tip (e); and selection of the different calli types (f).

\section{Callus induction}

Different calli types were obtained (hard and green, white and friable, yellow) and then selected and propagated. Cultivating on the 5 modified MS media described above yielded several calli types which were easily distinguishable on the basis of morphological appearance. Predominantly green and dense calli appeared on the medium MS 2,4D $(0.5 \mu \mathrm{M})+$ Kin $(10$ $\mu \mathrm{M})$ (Fig. 1d). Some yellow and dense calli were obtained on the media MS 2,4D $(0.5 \mu \mathrm{M})+\operatorname{Kin}(0.5 \mu \mathrm{M})$ and MS 2,4D $(0.5 \mu \mathrm{M})+$ Kin $(1 \mu \mathrm{M})$, but did not grow or proliferate well during these trials. Explants of one centimeter hypocotyl were the most responsive to callus induction on these media. On the other hand, white and friable calli were obtained on MS 2,4D $(0.5 \mu \mathrm{M})+\mathrm{K}(10 \mu \mathrm{M})$ with explants taken from seedling shoot tips (Fig. 1e). The efficacy of producing white calli was 100\%, i.e. all trials within these experimental conditions yielded viable white and friable calli. The last type of calli obtained in these trials, was white and soft calli appearing from crown and hypocotyl explants on the medium MS 2,4D $(4.5 \mu \mathrm{M})$.

Calli production occurred preferably from explants inoculated on the medium MS 2,4D $(0.5 \mu \mathrm{M})+$ Kin $(10$ $\mu \mathrm{M})$ and to a lesser extent on the media MS 2,4D $(0.5 \mu \mathrm{M})$ $+\operatorname{Kin}(0.5 \mu \mathrm{M})$ and MS 2,4D $(0.5 \mu \mathrm{M})+\operatorname{Kin}(1 \mu \mathrm{M})$. The medium MS 2,4D $(4.5 \mu \mathrm{M})$ did not produce any homogeneous or reproducible callus and all explants established on this medium became necrotic after a few weeks. Interestingly, MS Kin $(2.32 \mu \mathrm{M})$ induced an important proliferation of shoots from hypocotyl and epicotyl explants (Fig. 2c), with a higher speed and rate than that observed with the regeneration from leaves proposed by Mujib (11).
With the exception of root explants, all other explants yielded calli. Hypocotyls appeared to be the most responsive tissue for callus production (Fig. 1c), which had been observed in previous reports (3). Explants from seedling shoot tips also gave very good results, especially on the medium MS 2,4D $(0.5 \mu \mathrm{M})+$ Kin $(10 \mu \mathrm{M})$. We also observed that only explants from hypocotyls maintained calli production after a two-month cultivation period.

In addition, the explant size was of great importance since the smallest explants decayed rapidly before any callus initiation was observed. The explants origin was also found to be important since different proliferation rates and calli types were observed with explants prepared from different parts of the plants. No callus was ever observed from root explants, whereas all other explants sources yielded calli. Finally, the age parameter was also found to be determinant. Whatever the culture medium, explants made at the youngest developmental stages (stage 1 and 2 shown in Fig. 1a) induced calli only occasionally. Moreover such young explants were affected by a quick dieback. The most appropriate developmental stage for callus induction and proliferation was the seedling stage 3 (Fig. 1a), also called 'visible epicotyls stage'.

\section{Proliferation and sesquiterperne induction}

A lineage of solid green callus was obtained by sampling with a scalpel under sterile conditions a six-week-old callus induced from hypocotyl segments and repeating this experiment through several successive lineages (Fig. 1f). Such calli were particularly selected for the faculty of unlimited and stable growth on the initiation medium MS 2,4D $(0.5 \mu \mathrm{M})+$ Kin $(10 \mu \mathrm{M})($ Fig. 2a), and for the qualitative and quantitative composition in terpene compounds. Attempts to establish calli culture in liquid media were carried out according to Das et al. (5) and Dey (6) but no proliferation was observed with any of the callus types.
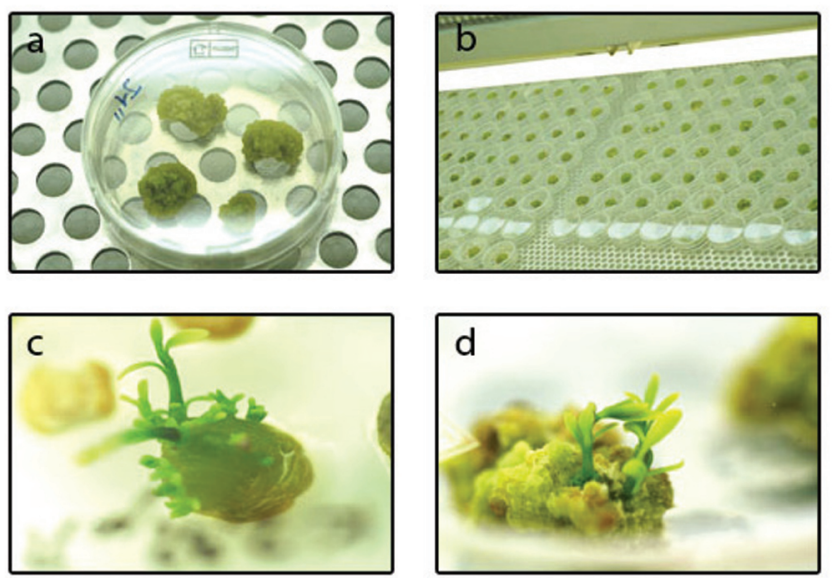

Fig. 2. Green calli selection and proliferation on $\mathrm{MS}+2,4 \mathrm{D}(0.5 \mu \mathrm{M})+\mathrm{Kin}$ $(10 \mu \mathrm{M})(\mathbf{a})$; calli library obtained from the green callus selected lineage (b); shoot proliferation from hypocotyl segments (c) and from green callus on $\mathrm{MS}+\operatorname{Kin}(2.5 \mu \mathrm{M})(\mathbf{d})$. 
Several culture media described above were evaluated to increase the proliferation rate. However, no useful improvement was observed. In some cases, for instance on medium MS 2,4D $(4.5 \mu \mathrm{M})$, the growth rate of the green callus was significantly higher but the resulting lineage turned finally into white and soft calli, or yellow and hard calli, with a lower terpene content and thus less favourable for the study the sesquiterpene metabolism.

In the described conditions, successive cultivation was maintained beyond 18 months on Gbg 2,4D $(0.5 \mu \mathrm{M})+\operatorname{Kin}(10$ $\mu \mathrm{M})$ and yielded a selected lineage library consisting of more than 200 individual calli (Fig. 2b). These calli were obtained by reproduction and successive divisions carried out every six weeks and were used for studying the terpene biosynthetic pathway by culture on inductive media and GC-MS.

In addition, the regeneration and the proliferation of axillary shoots on hypocotyl segments (Fig. 2c) and calli (Fig. 2d) were successfully obtained on the medium MS + Kin $(2.32 \mu \mathrm{M})$. The newly obtained shoots of Santalum album unfortunately failed to root despite many trials with different hormonal combinations on solid MS medium or by immersion in a naphthalene acetic acid (NAA) liquid medium as described in Sanjaya et al. (18). Although rooting was not obtained, these shoots proved to be usable as explants sources for further callus mass production.

\section{Chemical analysis}

Searching for terpenes and sesquiterpenes by GCMS analyses showed that selected calli were able to produce a range of terpenic molecules, including some desired terpenes important in the sandalwood odour profile such as beta-santalol, betasantalene and others listed in Fig. 3. Fig. 4 and Fig. 5 illustrate the capacity of in vitro tissues to produce terpene molecules constitutive of the sandalwood oil odour profile. Although the sesquiterpene profiles of calli differed from the commercial oil profiles in the relative quantities of the desired molecules, these molecules are synthesized by in vitro cultivated tissues, demonstrating that such tissues may be used for the identification of genes involved in the terpene metabolism, as achieved by Schalk (19) using the calli produced in the course of this study.

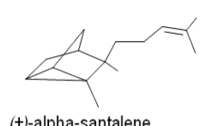

(+)-alpha-santalene
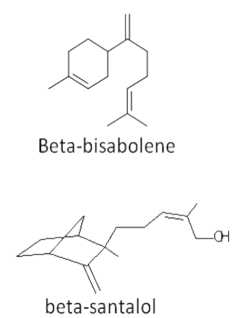
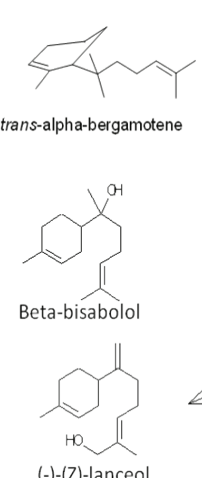

$(-)-(Z)-$ lanceol

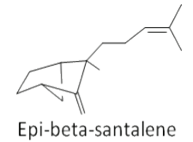

Epi-beta-santalene
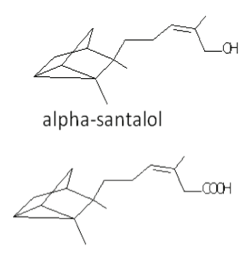

alpha-santalic acid

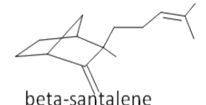

beta-santalene
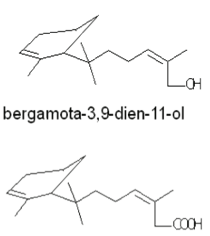

trans-alphabergamotenic-acid
Fig. 3. Some common terpenes of sandalwood oil.
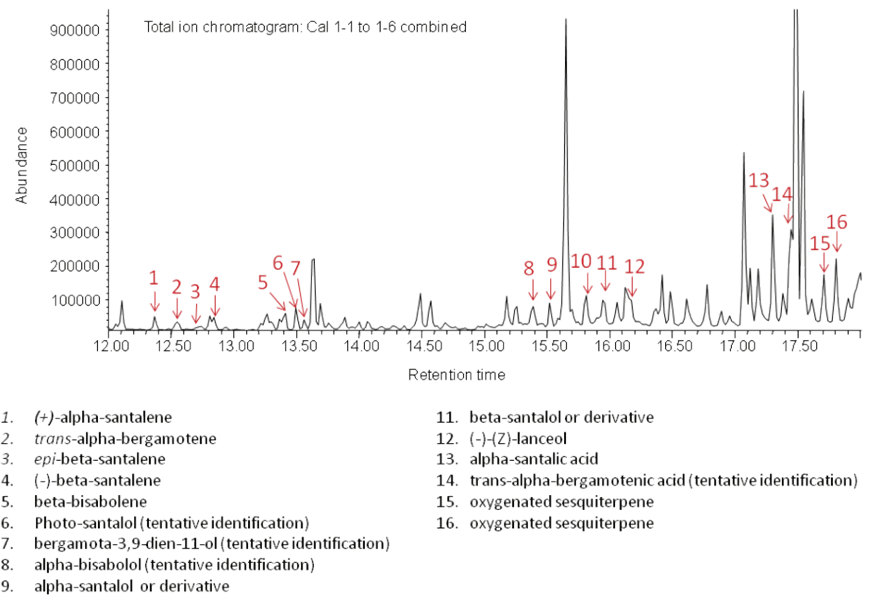

Fig. 4. GC-MS analysis of the composition in sesquiterpene compounds of induced calli growing on medium Gbg 2,4D $(0.5 \mu \mathrm{M})+\mathrm{K}(10 \mu \mathrm{M})+\mathrm{ACC}$ (1 mM).

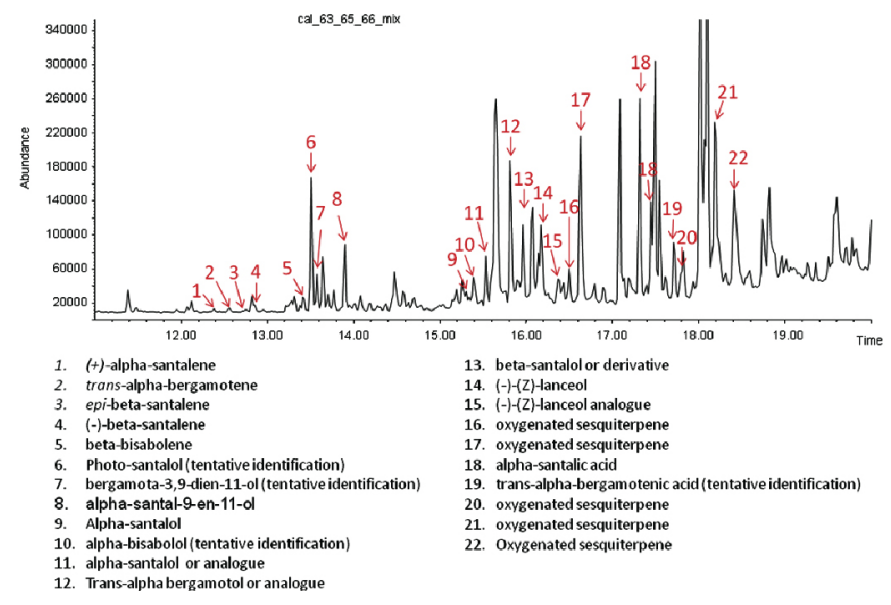

Fig. 5. GC-MS analysis of the composition in sesquiterpenes compounds of induced calli growing on medium Gbg K $(4.64 \mu \mathrm{M})+\mathrm{ACC}(2 \mathrm{mM})$. PIC ion contents $(\%)$ are indicated in brackets.

\section{Conclusions}

Several months of research led to the development of a protocol for in vitro production of sandalwood seedlings through in vitro germination with an average rate of $95 \%$ healthy sandalwood plants in culture. Our method represents a major improvement compared to the in vitro or in situ methods described so far for obtaining these plants.

The production of calli, especially green and dense calli, was shown to be faster and quantitatively more important on the medium MS 2,4D $(0.5 \mu \mathrm{M})+\operatorname{Kin}(10 \mu \mathrm{M})$ than on the four other tested media. The media MS 2,4D $(0.5 \mu \mathrm{M})+$ Kin $(0.5$ $\mu \mathrm{M})$ and MS 2,4D $(0.5 \mu \mathrm{M})+$ Kin $(1 \mu \mathrm{M})$ mainly induced yellow calli which did not proliferate. The medium MS 2,4D $(4.5 \mu \mathrm{M})$ did not produce any homogeneous or reproducible calli and all explants quickly decayed on this medium. Interestingly, the medium with kinetin alone did not induce any callus production but induced an important proliferation of adventitious shoots. This property was used for further biomass production using these adventitious shoots as explants. 
This work pointed out MS 2,4D $(0.5 \mu \mathrm{M})+\operatorname{Kin}(10 \mu \mathrm{M})$ and Gbg 2,4D $(0.5 \mu \mathrm{M})+\operatorname{Kin}(10 \mu \mathrm{M})$ as the most adequate media for the initiation and proliferation of Santalum album calli. In addition, concerning the choice of the explants, we observed that the visible epicotyls stage was the most appropriate developmental stage of the in vitro seedlings. Regarding explants responsiveness, the hypocotyl tissue proved to be the most reactive followed by the shoot tips. These two explants did not produce the same callus types.

Using the protocol described here, the in vitro culture of sandalwood plants and calli was maintained over a 3-year period, yielding a bank of over 200 calli from a single selected lineage. This material has been used in studies to isolate genes for santalol synthesis and to help elucidating the sesquiterpene metabolism in sandalwood. The results of this research led to the publication of a patent (19). Such a research program could allow biotechnological production of the desired aromatic compounds and consequently contribute to the preservation of this endangered species.

\section{Acknowledgements}

We are grateful to the Swiss Federal Commission for Technological and Innovation (CTI/KTI) and Firmenich S.A. for funding these works in the frame of the CTI Project No 9083.1 PFLS-LS.

\section{REFERENCES}

1. Ananthapadmanabha H.S. (2000) My Forest, 36, 147152.

2. Anonis D.P. (1998) Perfumer Flavorist, 23, 19-24.

3. Bapat V.A, Rao P.S. (1984) Plant Sci., 93(1), 19-22.

4. Bapat V.A., Rao P.S. (1985) Current Sci., 54, 978-982.

5. Das S., Pal S., Mujib A., Sahoo S.S., Dey S., Ponde N.R., Dasgupta S.A. (1999) Acta Hort., 502, 281-288.

6. Dey S. (2002) Sandalwood Research Newsletter (Australia), 13, 1-3.
7. Gamborg O.L., Miller R.A., Ojima K. (1968) Exp. Cell Res., 50(1), 151-158.

8. Howes M.-J. R., Simmons M.S.J., Kite G.C. (2004) J. Chrom. A, 1028(2), 307-312.

9. IUCN (2010) Red List of Threatened Species. Version 2010.4. Asian Regional Workshop (Conservation and Sustainable Management of Trees, Viet Nam) 1998. Santalum album.

10. Loneragan O.W. (1990) Research Bulletin No. 4. Department of Conservation and Land Management. Como, Perth.

11. Mujib A. (2005) Turk. J. Bot., 29, 63-67.

12. Murashige T., Skoog F.A. (1962) Physiol. Plantar., 15, 473-497.

13. Rao P.S., Bapat V.A., Mhatre M. (1984) Proc. Ind. Nat. Sci. Acad. Part B, 50(2), 196-202.

14. Rao P.S., Bapat V.A. (1992) In: Biotechnology in Agriculture and Forestry (Y.P.S. Bajaj, Ed.), SpringerVerlag, Berlin, 193-210.

15. Rao P.S., Bapat V.A. (1995) In: Somatic Embryogenesis in Woody Plants (S. Jain, P. Gupta, R. Newton, Eds.), Kluwer Academic Publishers, Dordrecht, 153-170.

16. Rugkhla A., Jones M.G.K. (1998) Exp. Bot., 49(320), 563-571.

17. Samantray S., Upadhyaya C. (2010) Int. J. Sci. Technol., 1(1), 10-18.

18. Sanjaya B.M., Thrilok S.R., Vittal R.R. (2006) J. For. Res., 11(3), 203-209.

19. Schalk M. (2010) Method for producing beta-santalene. International publication number WO/2010/067309 A1. World Intellectual Property Organisation International Bureau, Geneva.

20. Verghese J., Sunny T.P., Balakrishnan K.V. (1990) Flav. Fragr. J., 5(4), 223-226. 